\title{
FLOW EQUATIONS AND THEIR BORDERLINES FOR DIFFERENT REGIMES OF MASS TRANSFER
}

\author{
Jian $\mathrm{Li}^{1,2}$ and Yongbin Zhang ${ }^{1 *}$ \\ 1. College of Mechanical Engineering, Changzhou University, Changzhou,213164, Jiangsu Province, China \\ 2. Changzhou High Technology Research Key Laboratory of Mould Advanced Manufacturing, Changzhou, Jiangsu Province, China
}

\begin{abstract}
The paper introduces the flow equations for the fluid flows in a cylindrical tube respectively on the macroscale, multiscale and nanoscale, especially recently developed ones. It manifests that when these equations should be used in calculating the transferred mass and what should be taken into consideration when the tube inner radius is reduced to very small values. It gives an important indication on how to treat the mass transfer calculation for the tube flow on different size scales.

Keywords: Adsorbed layer; Continuum fluid; Flow; Multiscale; Nanoscale
\end{abstract}

\section{INTRODUCTION}

It is well known that the Hagen-Poiseuille equation can handle the flow problem in the cylindrical tube with macro size. However, when the inner radius of the tube was reduced to the scales of $1 \mathrm{~nm}$ or $10 \mathrm{~nm}$, it was found that the flow rate through the tube is much less or much greater than the classical Hagen-Poiseuille equation prediction (Calabrò et al., 2013; Myers, 2011; Sofos et al., 2015; Takaba et al., 2007); the former was attributed to the strong fluid-tube wall interaction, which results in the formation of the solidified layer on the tube wall (Sofos et al., 2015; Takaba et al., 2007) and the strong non-continuum effect of the fluid (Zhang, 2015); the latter was attributed to the weak fluid-tube wall interaction which results in the interfacial slippage on the tube wall (Calabrò et al., 2013; Myers, 2011). When the inner radius of the tube is on the scales of $10 \mathrm{~nm}$ or $100 \mathrm{~nm}$, the multiscale flow may occur in the tube, and it consists of the nanoscale non-continuum adsorbed layer flow and the intermediate continuum fluid flow (Chan and Horn, 1985); the surface property of the tube wall and the fluid-tube wall interaction have a significant influence on this multiscale flow, and the Hagen-Poiseuille equation fails for this flow. Chan and Horn (1985) experimentally observed the anomalous hydrodynamic lubrication behavior in a concentrated contact deviating from the classical Reynolds lubrication theory when the surface separation was below 50nm; They suggested the equivalent solid layer on the contact surface for satisfactorily explaining the anomalous phenomena. It is very possible that their observation be due to the multiscale hydrodynamic lubrication effect with the formation of the adsorbed layer on the contact surface, as they found that the hydrodynamic behavior still followed the classical Reynolds lubrication equation when the surface separation was over $50 \mathrm{~nm}$.

We have no difficulty in calculating the mass flow rate through the conventional cylindrical tube by using the classical hydrodynamic flow theories. But we often faced the difficulty in calculating the flow rate through the cylindrical tube with very small inner radii where the multiscale flow or even the nanoscale non-continuum flow occur; the popular dints for these calculations are to use molecular dynamics simulation (MDS) (Atkas and Aluru, 2002; Borg et al., 2013; Dang and Chang, 1997; Liu et al., 2007; Nie et al., 2004; Sun et al., 2010; Yang and Zheng, 2010; Yen et al. 2007). However, for engineering flows with large ratios of the tube axial length to the tube inner radius, the costs of computational time and computer storage are normally unaffordable with the use of MDS. We face the task to develop new calculation models for the mass transfer in the very small cylindrical tube with the multiscale or nanoscale flows.

This paper reviews the recent emerging theoretical models and calculation equations for the multiscale and nanoscale flows in the cylindrical tube. The validity of these equations is discussed. The borderlines among the calculation equations are shown. The paper emphasizes the effect of the adsorbed layer on the tube wall which should be taken into consideration when facing the flow problem in the cylindrical tube the inner radius of which is less than one hundred times of the thickness of the adsorbed layer.

\section{DIFFERENT FLOW REGIMES IN THE CYLINDRICAL TUBES WITH DIFFERENT TUBE INNER RADII}

The cylindrical tubes with different inner radii have applications in different areas. Their sizes are varied in wide ranges. In animals, the blood vessels including the capillary vessels should belong to the cylindrical tubes with macroscale sizes, where the blood flow is described by the continuum flow theory (Taylor et al., 1998). In conventional cooling and heating systems, the cylindrical tubes where the liquid media flows also belong to those with macroscale size. It is well recognized that the Hagen-Poiseuille equation can handle the flow in this sort of cylindrical tube. Figure 1(a) shows the cylindrical tube with the geometrical size $R_{0} / h_{b f} \geq 100$, where $R_{0}$ is the tube inner radius 
and $h_{b f}$ is the thickness of the adsorbed layer on the tube wall; actually, for the liquid flow in this type of cylindrical tube the continuum flow theory is valid ( $\mathrm{Li}$ and Zhang, 2021).

In micro/nano porous filtration membranes, the pore inner radius can be on the scales of $1 \mu \mathrm{m}, 100 \mathrm{~nm}$ and $10 \mathrm{~nm}$. In micro cooling and heating systems, the inner radius of the cylindrical tube can also be on such scales. In this type of cylindrical tube, the thickness $h_{b f}$ of the adsorbed layer on the tube wall becomes comparable to the tube inner radius $R_{0}$; the flowing media in such a tube is actually as Fig.1(b) shows; there may be several annular molecular layers physically adsorbed to the tube wall, while surrounded by the adsorbed layer is the continuum liquid. It was found that for $1<R_{0} / h_{b f}<100$, the flow in the cylindrical tube should be treated as multiscale by incorporating both the flow of the adsorbed layer and the flow of the continuum liquid ( $\mathrm{Li}$ and $\mathrm{Zhang}$, 2021).

In a super filtration membrane, the radius $R_{0}$ of the filtration pore can be on the scale of $1 \mathrm{~nm}$ and no more than the thickness $h_{b f}$ of the potentially formed adsorbed layer on the tube wall. In this case, the continuum liquid flow vanishes and the non-continuum flow occurs in the whole pore. The flow through the cell membrane in an animal may be also like this case. The non-continuum flow equation should solve this flow problem.

The wall slippage not only occurs in the macroscopic cylindrical tube as like Fig.1(a) shows but also easily occurs in the microscopic and nanoscopic cylindrical tubes as like Figs.1(b) and (c) show. It can improve the flow rate through the nanotube with two orders higher than the classical Hagen-Poiseuille equation prediction (Majumder et al., 2005; Mattia and Calabro, 2012; Li and Zhang, 2021). It was attributed to the hydrophobic tube wall and the weak fluid-tube wall interaction (Majumder et al., 2005; Mattia and Calabro, 2012).

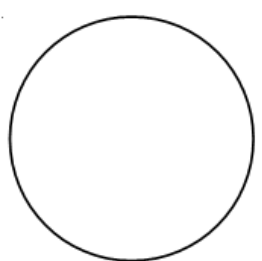

(a) $R_{0} / h_{b f} \geq 100$

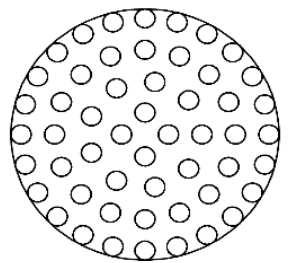

(c) $R_{0} / h_{b f} \leq 1$

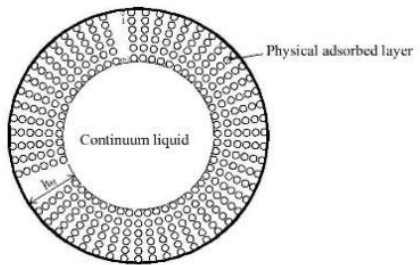

(b) $1<R_{0} / h_{b f}<100$

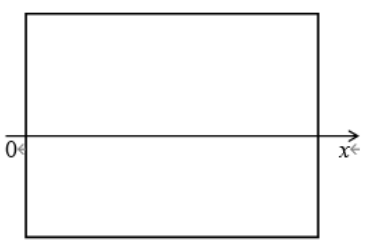

(d) Coordinate along the axial direction of the tube
Fig. 1 Cylindrical tubes with different flow regimes. (a) Continuum flow; (b) Multiscale flow; (c) Nanoscale non-continuum flow; (d) Axial profile of the cylindrical tube with the used coordinate.

\section{FLOW EQUATIONS FOR THE THREE FLOW REGIMES}

\subsection{For the continuum flow}

In the cylindrical tube shown by Fig.1(a), the thickness $h_{b f}$ of the adsorbed layer on the tube wall is far less than the tube inner radius $R_{0}$ and the effect of the adsorbed layer is negligible. Based on the assumptions of the Newtonian liquid and the laminar, isothermal and steady flow, accounting for the wall slippage, the continuum equation for calculating the mass flow rate $q_{m}$ (in $\mathrm{kg} / \mathrm{s}$ ) through the tube in Fig.1(a) is: $q_{m}=\pi \rho \bar{u} R_{0}^{2}-\frac{\pi \rho R_{0}^{4}}{4 \eta} \frac{\partial p}{\partial x}, \quad$ for $R_{0} / h_{b f} \geq 100$

where $\bar{u}$ is the slipping velocity of the liquid adjacent to the tube wall, $\rho$ and $\eta$ are respectively the bulk density and bulk viscosity of the liquid, $p$ is the pressure driving the flow, and $x$ is the coordinate along the axial direction.

In Eq (1), for no wall slippage, put $\bar{u}=0$; for the wall slippage occurrence, put $\partial p / \partial x=-\tau_{s, f-w} / R_{0}$, where $\tau_{s, f-w}$ is the interfacial shear strength on the fluid-tube wall interface, and the interfacial slipping velocity $\bar{u}$ is determined by the power loss on the whole tube.

\subsection{For the multiscale flow}

In the cylindrical tube shown by Fig.1(b), the molecules within the adsorbed layer are orientated (Pu and Liu, 1999) and the local density and local viscosity within the layer are varied (Bitsanis et al., 1987) due to the fluid-tube wall interaction. Both the effective viscosity and the average density of the layer are increased compared to the fluid bulk values (Meyer et al., 1998). Also, the combined effect of the discontinuity and inhomogeneity across the layer thickness should be considered (Zhang, 2006). The flow of the adsorbed layer should be described by the nanoscale non-continuum flow equation, and that of the continuum liquid should be described by the continuum flow equation. The flow in the tube is actually multiscale.

Calculating this multiscale flow is really challenging. We may use molecular dynamics simulation to calculate the flow rate of the adsorbed layer and use the continuum fluid model to calculate the flow rate of the continuum liquid (Atkas and Aluru, 2002; Borg et al., 2013; Dang and Chang, 1997; Liu et al., 2007; Nie et al., 2004; Sun et al., 2010; Yang and Zheng, 2010; Yen et al. 2007). This multiscale approach is hard to be implemented for an engineering multiscale flow, where the tube axial length, the tube thickness, and the circumferential length of the adsorbed layer area are often out of the range that MDS can model; The computational time and computer storage cost by MDS for the engineering multiscale flow is too large to be affordable.

\subsubsection{For the case of the adsorbed layer-tube wall interfacial slippage}

Based on the assumptions of the Newtonian continuum liquid and the laminar, isothermal and steady flow on the ensemble average, Zhang (2020a) developed a distinct multiscale approach for the multiscale flow in the tube in Fig.1(b), by using the equivalent continuum model to describe the adsorbed layer flow with incorporation of the above mentioned properties of the adsorbed layer. According to his approach, for the interfacial slippage on the adsorbed layer-tube wall interface, which may be due to the hydrophobic tube wall, the total mass flow rate through the tube in Fig.1(b) is calculated by the following equation (Zhang, 2020a):

$$
\begin{aligned}
& q_{m}=2 \pi R_{e, 0} \bar{u} h_{b f} \rho_{b f, 1}^{e f f}+\pi \bar{u}\left(R_{0}-h_{b f}\right)^{2} \rho \\
& +2 \pi R_{e, 0}\left[\frac{F_{1} h_{b f}^{3}}{12 \eta_{b f, 1}^{e f f}} \frac{\partial p}{\partial x}-\frac{h_{b f}^{3}}{2 \eta_{b f, 1}^{e f f}} \frac{\partial p}{\partial x}\right. \\
& \left.\cdot\left(1+\frac{1}{2 \lambda_{b f, 0}}-\frac{q_{0}-q_{0}^{n}}{q_{0}^{n-1}-q_{0}^{n}} \frac{\Delta_{n-2}}{h_{b f}}\right) \frac{\varepsilon}{1+\frac{\Delta x}{D}}\right] \rho_{b f, 1}^{e f f} \\
& +\left\{\frac{4}{\eta_{b f, 1}^{e f f}}\left[\frac{F_{2} \lambda_{b f, 0}^{2}}{6}-\frac{\lambda_{b f, 0}}{1+\frac{\Delta x}{D}}\left(\frac{1}{2}+\lambda_{b f, 0}-\frac{\left(q_{0}-q_{0}^{n}\right) \Delta_{n-2}}{2\left(q_{0}^{n-1}-q_{0}^{n}\right)\left(R_{0}-h_{b f}\right)}\right)\right]\right. \\
& \left.-\frac{1}{4 \eta}\right\} \cdot \pi \rho\left(R_{0-} h_{b f}\right)^{4} \frac{\partial p}{\partial x},
\end{aligned}
$$$$
\text { for } 1<R_{0} / h_{b f}<100 \text { (2) }
$$

where $\bar{u}$ is the interfacial slipping velocity on the adsorbed layer-tube wall interface, $D$ is the diameter of the fluid molecule, $R_{e, 0}=R_{0}(1-$ $\left.\lambda_{x} / 2\right), \lambda_{b f, 0}=\lambda_{x} /\left[2\left(1-\lambda_{x}\right)\right], \lambda_{x}=h_{b f} / R_{0}, \quad \rho_{b f, 1}^{e f f}$ and $\eta_{b f, 1}^{e f f}$ are respectively the average density and the effective viscosity of the 
adsorbed layer across the layer thickness, $\eta_{b f, 1}^{e f f}=D h_{b f} /[(n-1)(D+$ $\left.\left.\Delta_{x}\right)\left(\Delta_{l} / \eta_{\text {line }, l}\right)_{\text {avr }, n-1}\right), q_{0}=\Delta_{j+1} / \Delta_{j}$ and $q_{0}$ is constant, $\Delta x$ is the separation between the neighboring liquid molecules in the flow direction in the adsorbed layer, $\varepsilon=(2 D I+I I) /\left[h_{b f}(n-1)\left(\Delta_{l} /\right.\right.$ $\left.\left.\eta_{\text {line }, l}\right)_{a v r, n-1}\right] \quad, \quad F_{1}=\eta_{b f}^{e f f}\left(12 D^{2} \psi+6 D \phi\right) / h_{b f}^{3} \quad, \quad$ and $\quad F_{2}=$ $6 \eta_{b f}^{\text {eff }} D(n-1)\left(l \Delta_{l-1} / \eta_{\text {line }, l-1}\right)_{a v r, n-1} / h_{b f}^{2} ;$ Here, $\quad I=\sum_{i=1}^{n-1} i\left(\Delta_{l} /\right.$ $\left.\eta_{\text {line }, l}\right)_{a v r, i}, I I=\sum_{i=0}^{n-2}\left[i\left(\Delta_{l} / \eta_{\text {line }, l}\right)_{\text {avr }, i}+(i+1)\left(\Delta_{l} / \eta_{\text {line }, l}\right)_{a v r, i+1}\right] \Delta_{i}$, $\psi=\sum_{i=1}^{n-1} i\left(l \Delta_{l-1} / \eta_{\text {line }, l-1}\right)_{a v r, i} \quad, \quad \phi=\sum_{i=0}^{n-2}\left[i\left(l \Delta_{l-1} / \eta_{\text {line }, l-1}\right)_{\text {avr }, i}+\right.$ $\left.(i+1)\left(l \Delta_{l-1} / \eta_{\text {line }, l-1}\right)_{\text {avr }, i+1}\right] \Delta_{i} \quad, \quad i\left(\Delta_{l} / \eta_{\text {line }, l}\right)_{a v r, i}=\sum_{j=1}^{i} \Delta_{j-1} /$ $\eta_{\text {line }, j-1}, \quad i\left(l \Delta_{l-1} / \eta_{\text {line }, l-1}\right)_{a v r, i}=\sum_{j=1}^{i} j \Delta_{j-1} / \eta_{\text {line }, j-1}, \quad n$ is the equivalent number of the fluid molecules across the adsorbed layer thickness, $\eta_{\text {line } j-1}$ and $\Delta_{j-1}$ are respectively the local viscosity and the separation between the $j^{t h}$ and $(j-1)^{t h}$ molecules across the adsorbed layer thickness, and $j$ and $(j-1)$ are respectively the order numbers of the molecules across the adsorbed layer thickness shown in Fig.1(b).

When no wall slippage occurs, equation (2) is valid just by putting $\bar{u}=0$. When the adsorbed layer-tube wall interfacial slippage occurs, by using the limiting interfacial shear strength model, which interprets the interfacial slippage as the result of the interfacial shear stress exceeding the interfacial shear strength, equation (2) will be valid by putting the pressure gradient as:

$\frac{\partial p}{\partial x}=\frac{\tau_{s, b-w}}{R_{0}-h_{b f}+D(n-1)}$

where $\tau_{s, b-w}$ is the adsorbed layer-tube wall interfacial shear strength.

\subsubsection{For the case of the adsorbed layer-fluid interfacial slippage}

For a hydrophilic cylindrical tube wall, the interfacial slippage may first occur on the adsorbed layer-fluid interfacial slippage, while it is absent on the adsorbed layer-tube wall interface. According to the multiscale approach developed by Zhang (2020b), which is based on the assumptions of the Newtonian rheological behavior within the continuum liquid and the laminar, isothermal and steady flow on the ensemble average, when this type of interfacial slippage occurs, the total mass flow rate through the tube is: $q_{m}=q_{m, b f}+q_{m, h f}$, where $q_{m, b f}$ is the mass flow rate of the adsorbed layer through the tube and calculated by the following equation:

$$
\begin{aligned}
q_{m, b f}=2 \pi \rho_{b f, 1}^{e f f} R_{e, 0} & {\left[\frac { h _ { b f } ^ { 3 } } { 2 \eta _ { b f , 1 } ^ { e f f } } \frac { \tau _ { s , b - f } } { R _ { 0 } - h _ { b f } } \left(1+\frac{1}{2 \lambda_{b f, 0}}\right.\right.} \\
& \left.\left.-\frac{q_{0}-q_{0}^{n}}{q_{0}^{n-1}-q_{0}^{n}} \frac{\Delta_{n-2}}{h_{b f}}\right) \frac{\varepsilon}{1+\frac{\Delta x}{D}}-\frac{F_{1} h_{b f}^{3}}{12 \eta_{b f, 1}^{e f f}} \frac{\tau_{s, b-f}}{R_{0}-h_{b f}}\right]
\end{aligned}
$$

and $q_{m, h f}$ is the mass flow rate of the continuum fluid through the tube and calculated by the following equation:

$$
\begin{gathered}
q_{m, h f}=\frac{\pi \rho\left(R_{0}-h_{b f}\right)^{3} \tau_{s, b-f}}{4 \eta} \\
+\pi \rho\left(R_{0}-h_{b f}\right)^{2}\left[\frac{\Delta P O W}{\pi \Delta l \tau_{s, b-f}\left(R_{0}-h_{b f}\right)}\right. \\
-\frac{2 F_{2}\left(R_{0}-h_{b f}\right) \tau_{s, b-f} \lambda_{b f, 0}^{2}}{3 \eta_{b f, 1}^{e f f}}-\frac{4\left(R_{0}-h_{b f}\right) \tau_{s, b-f} \lambda_{b f, 0}}{\eta_{b f, 1}^{e f f}\left(1+\frac{\Delta x}{D}\right)}
\end{gathered}
$$

$$
\left.\cdot\left(\frac{1}{2}+\lambda_{b f, 0}-\frac{\left(q_{0}-q_{0}^{n}\right) \Delta_{n-2}}{2\left(q_{0}^{n-1}-q_{0}^{n}\right)\left(R_{0}-h_{b f}\right)}\right)\right]
$$

Here, $\tau_{s, b-f}$ is the interfacial shear strength on the adsorbed layerfluid interface, $\Delta l$ is the axial length of the tube, $\triangle P O W=P O W-$ $P O W_{c r}, P O W$ is the power loss on the tube with the axial length $\Delta l$, and $P O W_{c r}$ is the critical power loss on the tube with the axial length $\Delta l$ for initiating the interfacial slippage.

\subsubsection{Commenting remarks}

Various calculations from Eqs.(2), (4) and (5) gave physically reasonable results for different fluid-tube wall interactions, which were qualitatively agreeable with the experimental results (Zhang, 2020a,b). Nevertheless, equations (2), (4) and (5) need to be further validated by direction comparison with the experiments or the MDS results. These equations are presented here to show the substantial progress in calculating the multiscale mass transfer in micro/nano cylindrical tubes.

\subsection{For the nanoscale non-continuum flow}

In the cylindrical tube shown by Fig.1(c), the continuum fluid vanishes and the whole flow is non-continuum contributed by the fluid molecule dynamics; the interaction between the fluid molecules and the interaction between the fluid and tube wall molecules both should have important influences on the flow in the tube. Molecular dynamics simulation was widely used to theoretically study this nanochannel flow (Abraham 1978; Chauveteau, 1984; Horn et al., 1989; Jabbarzadeh et al., 1997; Sofos et al., 2013; Somers and Davis, 1992). It was found by MDS that both the local density and local viscosity of the fluid in this narrow tube are varied across the tube inner radius due to the fluid-tube wall interaction (Abraham 1978; Chauveteau, 1984; Horn et al., 1989; Jabbarzadeh et al., 1997; Sofos et al., 2013; Somers and Davis, 1992). The flow in this tube can greatly deviate from the classical continuum hydrodynamic flow theory in the conditions of the strong or weak fluid-tube wall interactions (Calabrò et al., 2013; Myers, 2011; Sofos et al., 2015; Takaba et al., 2007). Zhang (2015) showed that the Couette flow results in the mass flow rate through the nanotube equal to the continuum flow theory calculation, while the pressure driven Poiseuille flow component results in the magnitude of the mass flow rate through the nanotube significantly smaller than the classical Hagen-Poiseuille flow equation calculation owing to the significant non-continuum effect of the fluid in the conditions of the medium or strong fluid-tube wall interactions.

Based on the assumption of the laminar, isothermal and steady flow on the ensemble average, by incorporating the wall slippage and the dynamic and non-continuum effects of the fluid, Zhang (2017) gave the following equation for calculating the mass flow rate through the tube in Fig.1(c):

$$
\begin{aligned}
q_{m}=\pi \rho_{b f, 2}^{e f f}\left(R_{0}\right) \bar{u} R_{0}{ }^{2}+\frac{\pi \rho_{b f, 2}^{e f f}\left(R_{0}\right) S\left(R_{0}\right) R_{0}{ }^{4}}{4 \eta_{b f, 2}^{e f f}\left(R_{0}\right)} & \frac{\partial p}{\partial x}, \\
& \text { for } R_{0} / h_{b f} \leq 1
\end{aligned}
$$

where $\bar{u}$ is the wall slipping velocity, $\rho_{b f, 2}^{\text {eff }}$ and $\eta_{b f, 2}^{\text {eff }}$ are respectively the average density and the effective viscosity of the confined fluid across the tube radius, and $S$ is the parameter describing the discontinuity and inhomogeneity effects i.e. the non-continuum effect of the confined fluid. For no wall slippage, put $\bar{u}=0$; for the wall slippage occurrence, put $\partial p / \partial x=-\tau_{s, b-w} /[D(n-1)]$, and the wall slipping velocity $\bar{u}$ is determined by the power loss on the whole tube.

By the same method, Zhang (2016) also derived the flow equation for the nanoscale non-continuum flow in the nano slit pore, which has been validated by comparison with a lot of MDS results. Recently, Jiang and Zhang (2021) presented the direct matching results between Zhang's nanoscale non-continuum flow model and molecular dynamics simulation and showed very good quantitative agreements between these two approaches as shown in Figs.2(a) and (b). 


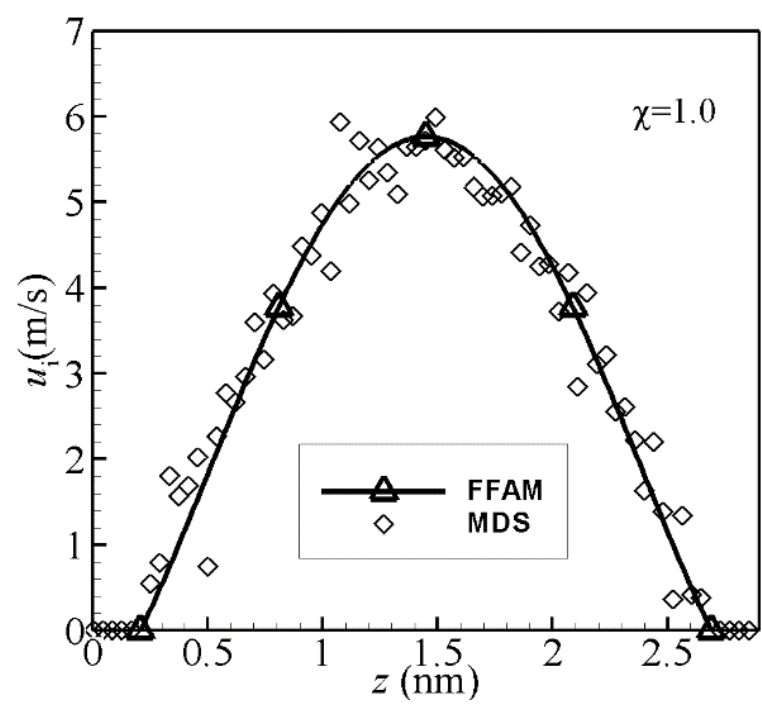

(a)

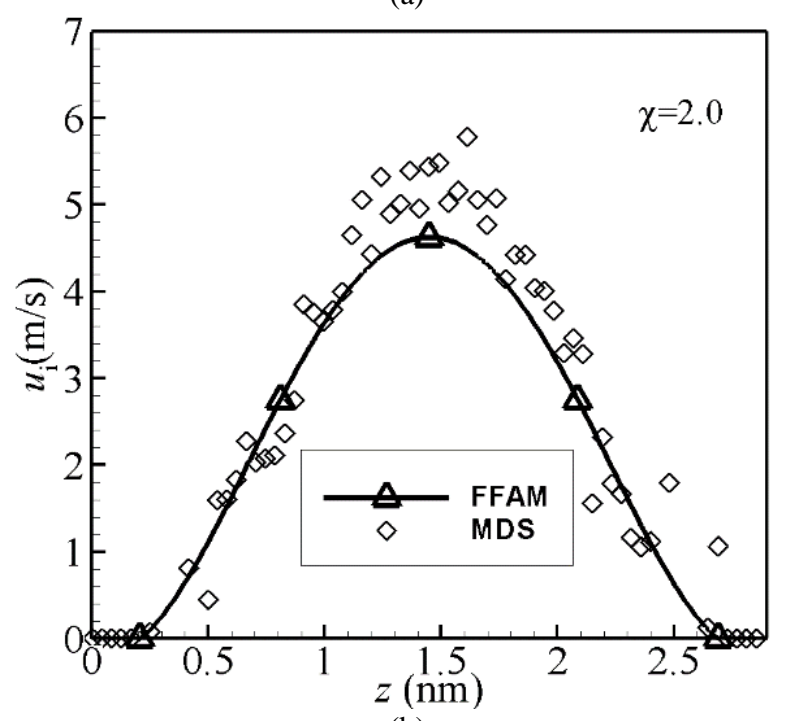

(b)

Fig. 2 Comparisons between Zhang's non-continuum flow model (FFAM) and molecular dynamics simulation (MDS) in the flow velocity profiles across the channel height for the flow in the nano slit pore, channel height $=2.898 \mathrm{~nm}, \chi$ is the factor of the interaction strength between the fluid and the pore wall (Jiang and Zhang, 2021).

\section{CONCLUSIONS}

Principally, there are three different flow regimes for the flow in a cylindrical tube, i.e. the continuum flow, the multiscale flow and the nanoscale non-continuum flow. Which flow regime prevails depends on the ratio $R_{0} / h_{b f}$ where $R_{0}$ is the tube inner radius and $h_{b f}$ is the thickness of the adsorbed layer on the tube wall. For $R_{0} / h_{b f} \geq 100$, the effect of the adsorbed layer is negligible and the continuum flow theory is applicable; for $1<R_{0} / h_{b f}<100$, the adsorbed layer effect should be considered, and the multiscale flow theory must be applied; for $R_{0} / h_{b f} \leq 1$, the continuum fluid vanishes and the whole flow is noncontinuum in the tube, and the nanoscale non-continuum flow theory must be used by incorporating the rheology evolution and the discontinuity and inhomogeneity effects of the fluid.

By incorporating the wall slippage or the adsorbed layer-fluid interfacial slippage, the flow equations are presented respectively for the above mentioned three flow regimes. Most of them have been substantiated, except that the multiscale flow equations need further verification by direct comparison with experiments (though the calculations from these equations showed physically reasonable results qualitatively agreeing with experiments).

The flow equations given in this paper have important implications for the modeling and calculation of the mass transfer in a cylindrical tube. They manifest that when we should use the corresponding flow equation for the flow in a cylindrical tube. These equations should have important application values in the studies of a lot of engineering problems such as blood vessel flows, micro/nano porous filtration membranes, flow through cell membranes and super purification.

\section{REFERENCES}

Abraham, F. F., 1978, “The interfacial density profile of a Lennard-Jones fluid in contact with a (100) Lennard-Jones wall and its relationship to idealized fluid/wall systems: A Monte Carlo simulation." J. Chem. Phys., 68, 3713-3716.

http://dx.doi.org/10.1063/1.436229

Atkas, O., Aluru, N. R., 2002, "A combined continuum/DSMC technique for multiscale analysis of microfluidic filters." J. Comput. Phys., 178, 342-372.

http://dx.doi.org/10.1006/jcph.2002.7030

Borg, M. K., Lockerby, D. A., Reese J. M., 2013, “A multiscale method for micro/nano flows of high aspect ratio.” J. Comput. Phys., 233, 400413.

http://dx.doi.org/10.1016/j.jcp.2012.09.009

Bitsanis, I., Magda, J. J., Tirrell, M., Davis, H. T., 1987, “Molecular dynamics of flow in micropores.” J. Chem. Phys., 87, 1733-1750. http://dx.doi.org/10.1063/1.453240

Calabrò, F., Lee, K. P., Mattia, D., 2013, "Modelling flow enhancement in nanochannels: Viscosity and slippage." Appl. Math. Lett., 26, 991994.

https://doi.org/10.1016/j.aml.2013.05.004

Chan, D. Y. C., Horn, R. G., 1985, "The drainage of thin liquid films between solid surfaces.” J. Chem. Phys., 83, 5311-5324.

https://doi.org/10.1063/1.449693

Chauveteau, G., Tirrell, M., Omari, A., 1984, "Concentration dependence of the effective viscosity of polymer solutions in small pores with repulsive or attractive walls." J. Coll. Interface Sci., 100, 41-54. https://doi.org/10.1016/0021-9797(84)90410-7

Dang, L. X., Chang, T. M., 1997, "Molecular dynamics study of water clusters, liquid and liquid-vapor interface of water with many body potentials.” J. Chem. Phys., 106, 8149-8154.

http://dx.doi.org/10.1063/1.473820

Horn, R. G., Smith, D. T., Haller, W., 1989, "Surface forces and viscosity of water measured between silica sheets." Chem. Phys. Lett., 162, 404408.

https://doi.org/10.1016/0009-2614(89)87066-6

Jabbarzadeh, A., Atkinson, J. D., Tanner, R. I., 1997, "Rheological properties of thin liquid films by molecular dynamics simulations." $J$. Non-Newtonian Fluid Mech., 69, 169-193.

http://dx.doi.org/10.1016//S0377-0257(96)01520-0

Jiang, C. T., Zhang, Y. B., 2021, "Direct matching between the flow factor approach model and molecular dynamics simulation for nanochannel flows." Int. Commun. Heat Mass Transf., submitted. 
Li, J., Zhang, Y. B., 2021, "Mass transfer in the filtration membrane covering from macroscale, multiscale to nanoscale." Membr. Water Treat., submitted.

Liu, J., Chen, S., Nie, X., Robbins, M. O., 2007, “A continuum-atomistic simulation of heat transfer in micro- and nano- flows." J. Comput. Phys., 227, 279-291.

http://dx.doi.org/10.1016/j.jcp.2007.07.014

Majumder, M., Chopra, N., Andrews, R., Hinds, B. J., 2005, "Enhanced flow in carbon nanotubes." Nature, 438, 44.

https://doi.org/10.1038/438044a

Mattia, D., Calabro, F., 2012, "Explaining high flow rate of water in carbon nanotubes via solid-liquid molecular interactions." Microfluid. Nanofluid., 13, 125-130.

https://doi.org/10.1007/s10404-012-0949-z

Meyer, E., Overney, R. M., Dransfeld, K., Gyalog, T., 1998, "Nanoscience-Friction and Rheology on the Nanometer Scale." World Scientific, New Jersey.

Myers, T. G., 2011, "Why are slip lengths so large in carbon nanotubes?" Microfluid. Nanofluid., 10, 1141-1145. http://dx.doi.org/10.1007/s10404-010-0752-7

Nie, X. B., Chen, S., Robbins, M. O., 2004, “A continuum and molecular dynamics hybrid method for micro- and nano- fluid flow." J. Fluid Mech., 500, 55-64.

http://dx.doi.org/10.1017/s0022112003007225

Pu, X. Y., Liu Q., 1999, “The theoretical study of Raman imaging for pendent drop adsorbed by uniaxial orientated molecules." Spectroscopy Spec. Anal., 19, 318-322.

http://dx.doi.org/10.1016/S1386-1425(99)00035-9

Sofos, F., Karakasidis, T. E., Liakopoulos, A., 2013, "Fluid flow at the nanoscale: How fluid properties deviate from the bulk." Nanosci. Nanotech. Lett., 5, 1-4.

http://dx.doi.org/10.1166/nnl.2013.1555

Sofos, F., Karakasidis, T. E., Liakopoulos, A., 2015, "Fluid structure and system dynamics in nanodevices for water desalination." Desalin. Water Treat., 55, 1-11.

http://dx.doi.org/10.1080/19443994.2015.1049966

Somers, S. A., Davis, H. T., 1992, "Microscopic dynamics of fluids confined between smooth and atomically structured solid surfaces." $J$. Chem. Phys., 96, 5389-5407.

\section{http://dx.doi.org/10.1063/1.462724}

Sun, J., He, Y., Tao, W. Q., 2010, "Scale effect on flow and thermal boundaries in micro-/nano- channel flow using molecular dynamicscontinuum hybrid simulation method." Int. J. Num. Meth. Eng., 81, 207228.

http://dx.doi.org/10.1002/nme.2683

Takaba, H., Onumata, Y., Nakao, S., 2007, "Molecular simulation of pressure-driven fluid flow in nanoporous membranes." J. Chem. Phys., 127, 054703.

http://dx.doi.org/10.1063/1.2749236

Taylor, C. A., Hughes, T. J. R., Zarins, C. K., 1998, "Finite element modeling of blood flow in arteries." Comput. Meth. Appl. Mech. Eng., 158, 155-196.

http://dx.doi.org/10.1016/S0045-7825(98)80008-X

Yang, X., Zheng, Z. C., 2010, "Effects of channel scale on slip length of flow in micro/nano channels." ASME J. Fluids Eng., 132, 061201. http://dx.doi.org/10.1115/1.4001619

Yen, T. H., Soong, C. Y., Tzeng, P. Y., 2007, "Hybrid molecular dynamics-continuum simulation for nano/mesoscale channel flows." Microfluid. Nanofluid., 3, 665-675. http://dx.doi.org/10.1007/s10404-007-0154-7

Zhang, Y. B., 2006, "Flow factor of non-continuum fluids in onedimensional contact." Industr. Lubr. Trib., 58, 151-169. http://dx.doi.org/10.1108/00368790610661999

Zhang, Y. B., 2015, "The flow factor approach model for the fluid flow in a nano channel." Int. J. Heat Mass Transf., 89, 733-742. https://doi.org/10.1016/j.ijheatmasstransfer.2015.05.092

Zhang, Y. B., 2016, "The flow equation for a nanoscale fluid flow." Int. J. Heat Mass Transf., 92, 1004-1008. https://doi.org/10.1016/j.ijheatmasstransfer.2015.09.008

Zhang, Y. B., 2017, “Transport in nanotube tree." Int. J. Heat Mass Transf., 114, 536-540.

http://dx.doi.org/10.1016/j.ijheatmasstransfer.2017.06.105

Zhang, Y. B., 2020a, "Modeling of flow in a micro cylindrical tube with the adsorbed layer effect: Part I-Results for no interfacial slippage." Int. J. Heat Mass Transf., submitted.

Zhang, Y. B., 2020b, "Multiscale analysis of micro/nano tube flow with the adsorbed layer-fluid interfacial slippage." Int. Commun. Heat Mass Transf., submitted. 